\title{
Editorial: OJPHI Vol 5, No 2 (2013)
}

Welcome to the current issue of the Online Journal of Public Health Informatics. At this stage I will like to take the opportunity to thank the reviewers who have been doing a wonderful job in providing prompt reviews of the articles assigned to them. We have achieved our status as a PubMed indexed, peer-reviewed journal mainly because you have taken this 'labor of love' assignment quite seriously. We plan to give graduate students an opportunity to submit summaries of their capstone reports and dissertations for publication as working papers in future issues of the journal. Authors will also be invited to deliver webinars on their articles to the public health informatics community. These webinars and their accompanying discussions will be recorded and archived. Students will be encouraged to attend these webinars. It is fair to say that, through the support of the reviewers and readers we have succeeded in positioning this journal as the sole portal for disseminating public health informatics research findings.

The capability to exchange patient-specific health data among autonomous healthcare entities is at the core of successful implementation of Health Information Exchanges. This capability is also important for patient treatment, public health services delivery, and research. Wu Xu et al. describe efforts to create a statewide Master Person Index in Utah to uniquely identify each individual receiving healthcare or public health services. Exchanging personally identifiable information across enterprises for healthcare identity resolution requires new models for data sharing and a complex policy framework to mitigate risks to participants and ensure cooperative success. The authors developed a focus area maturity model to guide the complex process of developing a functional statewide Master Person Index (sMPI) among diverse, autonomous partners. The proposed framework provides an orderly path to address interdependences that can guide the complex process of developing a functional sMPI, avoiding conflicts between policy and technology that may lead to nonfunctional implementations.

Immunization registries have been shown to increase vaccine coverage rates and reduce duplicate immunizations. In order to achieve meaningful use the Health Information Technology for Economic and Clinical Health (HITECH) Act of 2009 encourages providers to submit electronic immunization data to regional or statewide immunization information systems. At present, many providers have established unidirectional interoperability for uploading EMR immunization data to designated registries. It is recognized that bidirectional interoperability is preferable because it allows immunization data to be transmitted directly to vendor EMRs. Integration is, however, expensive and difficult to achieve. Lindsay A. Stevens, Jonathan P. Palma, et.al develop and test the feasibility of visually integrating external registries into vendor EMR systems. The study shows that this procedure meets providers' need for relevant data, increases reporting of immunizations, improves provider satisfaction, and avoids the increased costs of bidirectional data integration.

Approximately 300,000 individuals die from out-of-hospital cardiac arrest (OHCA) annually in the United States. It has been observed that there is a wide disparity in the OHCA survival rates between cities in USA. Hugh Semple et al. present results from an on-going project to develop user-friendly, interactive web mapping application that allows public health professionals and the general public to visualize the geographic patterns of cardiac arrest rates, bystander CPR rates, and target specific locations for public health services delivery. Participants in a preliminary 
evaluation felt that the web mapping application was a useful, user-friendly geovisualization tool. It is expected that this project will encourage the development of public health web mapping applications that are centered on interactive maps, summary statistics, and the use of social media technology.

Various social and institutional issues present challenges in the implementation of school-based sexual health education in developing countries. Angella Musiimenta of Mbarara University of Science and Technology, Uganda, presents the results of a study aimed at identifying the factors responsible for successful implementation of technology-aided sexual health education in two Ugandan schools. The results indicate that, rather than focusing exclusively on technology, program administrators should create social, institutional, and religious climate that is more supportive of school-based computer-assisted education.

Health services and public health researchers increasingly rely on search engines to identify relevant articles. The reliability of these search engines is very necessary if one is to avoid costly mistakes. Google Scholar (GS) is widely-recognized as an excellent source of grey literature in biomedicine. It is a useful tool to help researchers quickly locate relevant papers from billions of pages across the Web. Research by Dean Guistini et al. demonstrates that GS is not flexible, precise or indexed enough to be used alone for systematic reviews. The authors show that Google Search's 'keyword search' capability, allied to Google's PageRank, is a poor replacement for controlled vocabulary searching and its interface does not provide enough flexibility to accommodate search filters by discipline, such as 'health and medicine'. The authors recommend that Google Search developers should provide full details about its database coverage and improve its interface search capabilities (e.g., indexing, semantic search filters, stored searching, etc.) in order to satisfy the demands of thorough, replicable searches as required by systematic reviews for health services and public health research.

Thomas G. Savel et al. describe the development of Partial Thromboplastin Time (PTT) Advisor, a CDC-supported initiative to develop a mobile clinical laboratory decision support application. This is among the first of a handful of iOS-based applications funded by CDC. The application offers clinicians a resource to quickly select the appropriate follow-up tests to evaluate patients with prolonged PTT and a normal prothrombin laboratory result. The authors address some of the challenges involved in the development and deployment of the application. The free mobile PTT Advisor app was approved by Apple and published in their iTunes App store. Lessons learned from this project will assist other mobile health/public health application developers understand and overcome some of the challenges involved in such projects.

The determination of priorities is an essential component of community health status assessment. Priority setting enables the rational allocation of limited resources among competing programs. James Studnicki et al. utilized the analytical capabilities of online analytic processing( OLAP) interface to create a community health status prioritizing system which, among other attributes, is capable of ranking different types of health status outcomes and also provides flexibility in the weighting of the evaluation criteria. The authors demonstrate that rankings of community health outcomes based on OLAP provide sufficient information for priority setting compared to previous methods based on a static set of criteria with fixed weighting factors. 
The adoption of certified electronic health records and the implementation of health information exchanges are expected to facilitate the sharing of patients' health records by authorized providers. Records can thus follow patients resulting in reduced delays, duplications, errors, quality improvements and lower costs. Ultimately, the idea is to enable the patient to control access to their own data. Access to care records is currently often difficult or impossible in cases where records contain personal identifiers; they have to be secured behind services that greatly impede ready access. Existing access control infrastructures are proprietary, further making it impossible to retrieve patient records electronically on demand from a workstation that is not part of the record keeper's network. Existing solutions to the data portability problem have raised issues as to whether the public would have confidence that their personal records were safe, secure and private, especially when cloud-based or controlled by third party commercial service providers such as MicrosoftVault, GoogleHealth, etc. Roderick Neame outlines a platform-independent method that avoids most of the issues raised by existing record portability solutions and ensures continuum of care for patients such that their care records follow them wherever they go. In order for such a methodology to be implemented successfully the author acknowledges that it is necessary to have an agreement on the record data types and sub-types, their associated XML tags, as well as develop a browser add-on that can import and display flexibly the XML marked-up records. These conditions are not difficult to achieve with existing technologies.

Research shows that the incidence of healthcare- associated infections (HAIs) has increased significantly in the past 20 years in USA. The number of patients who suffer from HAIs annually in USA is estimated to be approximately 2 million, with about 100,000 deaths annually, ranking HAI among the leading causes of death in US acute-care hospitals. The federal government has mandated hospitals to publically report HAI rates in order to increase transparency and trust between hospitals and consumers, and to disseminate best practices. However, there is limited guidance in the medical and public health literature related to public reporting of health careassociated infections data. Yair Rajwan et al. demonstrate that visual communication can provide effective evidence-based information to consumers for decision making and to practitioners for providing patient safety outcomes and processes.

The prevention of hospital readmissions improves the quality of individual care as well as population health status. Under the Hospital Readmissions Reduction Program hospitals must reduce readmissions in order to avoid being penalized financially. Accurately predicting the risks of readmissions is a requirement for improving the transition of care process during and postdischarge. The use of administrative claims data is a major limitation of most risk prediction models. Shahid Choudhry, Jing Li et al. utilized electronic health records data and a mixedmethod risk prediction model to evaluate post-discharge risk factors. The model demonstrated reasonable fit in heterogeneous populations. Given the range of variables that contribute to readmission risks it is necessary to include variables from electronic health records in developing hospital readmission risks.

Noncomunicable diseases ( NCD) currently constitute the leading causes of deaths in all regions of the world except Africa. The largest increase in NCD deaths by 2020 is projected for Africa, Eastern Mediterranean and South-East Asian countries. Stephan Kohler reviews web portals that provide information for reducing preventable lifestyle-related risk factors associated with NCDs. 
The author also discusses an open access web portal initiated by two German states for NCD prevention and health promotion activities.

This issue includes two commentaries. The first commentary addresses the sustainability of public health surveillance systems. In recent years the world has witnessed disease outbreaks and epidemics resulting in loss of lives and significant economic costs. For example, the global Severe Acute Respiratory Syndrome of 2002-2003 resulted in a financial cost of $\$ 40$ billion to $\$ 54$ billion dollars while the anthrax attack in US in 2001 resulted in financial cost of 320 million dollars, 22 cases, including 5 deaths. The significant health impacts and economic costs of disease outbreaks illustrate the critical importance of effective public health surveillance and rapid response. In order to respond effectively to the growing threats to population health, public health surveillance systems must be built on a stable infrastructure of core workforce competencies, information systems, and organizational capacity, and must be supported by enterprise-based funding. Nabila Mirza, Terra Reynolds et al. present the recommendations of the Sustainable Surveillance Workgroup convened by the International Society for Disease Surveillance to identify strategies for building, strengthening, and maintaining surveillance systems.

A disparity currently exists between the accuracy of ICD-9 admission coding and discharge coding with some error rates as high as seventy percent. It is envisioned that the transition to ICD-10 coding could increase this disparity. In the second commentary, Christopher Bell, Arash Jalali, et.al. propose a decision support technology, the ICD-10 anatomographer, which could assist emergency department physicians working in busy trauma units in finding accurate ICD10 codes efficiently, thereby improving quality of care.

Best Regards

Edward Mensah, PhD

Editor-in-Chief

Online Journal of Public Health Informatics

1603 West Taylor Street, Room 759

Chicago, Illinois, 60612

Email:dehasnem@uic.edu

Office: 312-996-3001 